\title{
Onion-skin method (OSM) analysis of DIII-D edge measurements
}

\author{
P.C. Stangeby, ${ }^{\text {a }}$ J.G. Watkins, ${ }^{\text {b G.D. Porter, }}{ }^{\mathrm{c}}$ J.D. Elder, ${ }^{\mathrm{a}}$ S. Lisgo, ${ }^{\mathrm{a}}$ D. Reiter, ${ }^{\mathrm{d}}$ W.P. West, ${ }^{\mathrm{e}}$ \\ and D.G. Whytef \\ ${ }^{a}$ University of Toronto Institute for Aerospace Studies, 4925 Dufferin St, Toronto, M3H 5T6, Canada

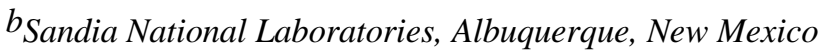 \\ ${ }^{c}$ Lawrence Livermore National Laboratory, Livermore, California \\ $d_{\text {University off Duesseldorf, Duesseldorf, Germany }}$ \\ e General Atomics, P.O. Box 85608, San Diego, CA 92186-5608 \\ $f_{\text {University of California, San Diego, California }}$
}

\begin{abstract}
OSM analysis provides, in principle, a method for establishing the 2-D edge "fields" of $\mathrm{n}_{\mathrm{e}}$, $T_{e}, T_{i}$, etc, which is the prerequisite for analyzing the physics processes occurring in the edge, including impurity behavior. In order to further test this method, an OSM analysis of an extensive edge database for an L-mode DIII-D discharge has been carried out, the first part of which is reported here. Consistency of the OSM results with Langmuir probe, $\mathrm{D}_{\alpha}$, and edge Thomson scattering measurements encourages further development of the method.
\end{abstract}

Keywords: SOL plasma; divertor plasma; DIII-D; 2D model; OSM model; fluid simulation.

\section{Introduction}

For the confined plasma, the measurement of $n_{e}(r), T_{e}(r), T_{i}(r)$ has always been considered to be of paramount importance. Without such spatially detailed information it would be difficult to 
understand what is happening in the main plasma. With the possession of such information it is possible to carry out detailed analysis of the processes occurring there, including identification of the cross-field transport coefficients, etc. In order to understand what is happening in the edge plasma it is probably equally necessary to know the 2-D "fields" of $n_{e}, T_{e}, T_{i}$ (and also the parallel flow velocity, $\mathrm{v}_{\|}$, and the plasma potential, V). Possession of such spatially detailed information would, in principle, make it possible to analyze the physical processes occurring in the edge in an undertaking that could parallel that made for the main plasma. For example, Monte Carlo codes — both for hydrogen neutrals, e.g. EIRENE [1], and impurity neutral+ion, production+transport codes, e.g. DIVIMP [2] — can be run using the 2-D edge fields as "plasma background" with the purpose of understanding hydrogen and helium pumping, the sources and transport of impurities, etc.

In contrast with the 1-D main plasma fields, the 2-D edge fields are not fully measured on any tokamak and some means — inevitably involving modeling — is required in order to piece the edge picture together from the variety of edge measurements made at various locations. In principle Onion-Skin Method (OSM), analysis [3,4] is one way of carrying out this undertaking. It is a method, however, that has only been tested to a limited degree to date. On JET, OSM analysis was recently applied to a collection of discharges, with boundary condition input taken from Langmuir probes, built into the divertor targets; the only code-experiment comparisons were made upstream at the location of a fast reciprocating probe [5]. The present paper reports on the first part of a more extensive confrontation of OSM analysis and edge experimental data than has been attempted so far - exploiting the comprehensive DIII-D edge measurements. The focus here, as in the JET study, is on the "plasma background," i.e. the hydrogen-related measurements. DIVIMP impurity analysis will be reported on later. Also as in the JET study, the OSM analysis here is restricted to the attached outer divertor SOL; analysis of the (usually) detached inner divertor SOL will be a separate study. The private flux zone poses particular problems for analysis and will also be examined separately. 
OSM analysis provides a complimentary method to standard 2-D fluid codes, such as UEDGE [6], for modeling edge plasmas. In standard 2-D codes, "point" boundary conditions, density and power input, are set at the "upstream end"; the values of the cross-field transport coefficients, $\mathrm{D}_{\perp}^{\mathrm{SOL}}, \chi_{\perp}^{\mathrm{SOL}}$, must also be specified. In OSM analysis a larger amount of experimental information is input, namely "line" boundary conditions spanning across the SOL - typically target probe measurements of $\mathrm{T}_{\mathrm{e}}$ and $\mathrm{I}_{\text {sat }}^{+}$as a function of distance across the targets, which is necessary and sufficient to constrain OSM solutions. In OSM analysis, the plasma edge region is divided up into nested, along-B, computational 'rings' ("onion-skins") and the plasma conservation equations are solved along each 'ring' using the experimental boundary conditions at the targets. The values of $\mathrm{D}_{\perp}^{\mathrm{SOL}}, \chi_{\perp}^{\mathrm{SOL}}$, rather than being an input, can be extracted as part of the OSM analysis. OSM analysis can be carried out at various levels of sophistication. In the most basic form, the standard divertor Two-point model [7] is applied to each SOL flux tube of the computational grid, thus giving the plasma parameters at each point upstream of the target, i.e. a 2-D solution is generated. OSM solutions are inherently 2-D since cross-field transport is necessarily included (although since virtually all of the computational effort is focused on solving the variations of plasma quantities along the field lines, it is sometimes thought incorrectly that it is 1-D). In the OSM used here, essentially the same parallel and atomic physics is used as that in the standard 2-D fluid edge codes. The usual conservation equations are solved iteratively with a Monte Carlo neutral code, here EIRENE, which provides the volumetric terms such as the ionization rate, needed in the OSM's conservation equations. At the end of each iteration the overall balances (of particles, momentum and energy) for each flux tube are computed and thus the total cross-field sources or sinks for the flux tube are known. The spatial distribution of the cross-field fluxes, the only free element, can be made proportional to $\mathrm{d}^{2} \mathrm{n} / \mathrm{dr}^{2}$, etc., to give diffusive transport, but OSM solutions are rather insensitive to the spatial distributions when working from "downstream" boundary conditions, e.g. target probe data. This is a beneficial feature of OSM analysis since cross-field transport is anomalous and it is not 
actually known if transport coefficients are spatially constant or not — or even if the transport is diffusive. 


\section{OSM analysis of a DIII-D L-mode discharge}

DIII-D shot no. 86575 was a lower single-null, L-mode discharge during 1 to $2 \mathrm{~s}$, subject to neutral beam heating of $0.85 \mathrm{MW} ; \mathrm{R}_{\mathrm{o}}=1.7 \mathrm{~m}, \mathrm{a}=0.6 \mathrm{~m}, \kappa=1.75, \mathrm{I}_{\mathrm{p}}=0.9 \mathrm{MA}, \mathrm{P}_{\Omega}=0.58 \mathrm{MW}$, $\mathrm{P}_{\mathrm{rad}}=0.87 \mathrm{MW}, \mathrm{n}_{\mathrm{e} 0}=2.1 \times 10^{19} \mathrm{~m}^{-3}$ (density on axis), $\mathrm{B}_{\phi}=2.1 \mathrm{~T}, \mathrm{q} 95=5.6$, ion B-drift toward the divertor. A divertor Thomson scattering system [8] provided measurements of $n_{e}$ and $T_{e}$ in the divertor region along a vertical line at $\mathrm{R}=1.49 \mathrm{~m}$, from just above the divertor floor to a height of $20 \mathrm{~cm}$ (see Fig. 1). In order to obtain Thomson data throughout the divertor region, the $\mathrm{X}$-point was swept; the outer strike point was moved (approximately smoothly) from $\mathrm{R}=1.63 \mathrm{~m}$ at $1.2 \mathrm{~s}$ to $\mathrm{R}=1.5 \mathrm{~m}$ at $1.7 \mathrm{~s}$. An array of probes built into the divertor targets [9] provided measurements of $\mathrm{T}_{\mathrm{e}}$ and $\mathrm{I}_{\text {sat }}^{+}$across the outer target. The poloidal distribution of the emissivity of $\mathrm{D}_{\alpha}$ light across the divertor was measured using an absolutely calibrated photodiode array, "filterscope", Fig. 1. The Upstream Thomson scattering system provided measurements of $\mathrm{n}_{\mathrm{e}}$ and $\mathrm{T}_{\mathrm{e}}$ across the SOL and into the confined plasma [8], Fig. 1. The probe profiles of $\mathrm{T}_{\mathrm{e}}$ and $\mathrm{I}_{\text {sat }}^{+}$ across the outer target are shown in Fig. 2.

Further details of the OSM model used here: $\mathrm{T}_{\mathrm{i}}=\mathrm{T}_{\mathrm{e}}$ at target assumed; sonic target assumed. The hydrogenic radiative cooling was computed using EIRENE; it was then tripled as an approximate way to account for impurity cooling $\left(\mathrm{P}_{\mathrm{rad}}^{\mathrm{C}} \approx 2 \mathrm{P}_{\mathrm{rad}}^{\mathrm{D}}\right.$ for DIII-D L-mode discharges [10]). EIRENE included neutral-neutral collisions (D-D, D-D $2, \mathrm{D}_{2}-\mathrm{D}_{2}$ ) within the plasma regions (but not yet throughout all of the non-plasma regions), also $\mathrm{D}^{+}-\mathrm{D}_{2}$ collisions. Power loss from outer SOL to private zone was included in an approximate way by calculating power loss from the SOL based on the power to the private part of the target.

Magnetic equilibria for various times in $1.2-1.7 \mathrm{~s}$ were obtained using the EFIT code [11] and computational grids were generated. These were largely orthogonal grids but were nonorthogonal near the targets to conform to the actual target shape. The OSM analysis was for the conditions at $1.65 \mathrm{~s}$ when the outer strike point was placed approximately on the Thomson 
location, $\mathrm{R}=1.49 \mathrm{~m}$ (the strike point location is only identified to within $\sim \pm 1 \mathrm{~cm}$ by EFIT, due to its uncertainties). Experimental data, e.g. Thomson values of $\mathrm{n}_{\mathrm{e}}$ and $\mathrm{T}_{\mathrm{e}}$, within a time-window of $\pm 0.1 \mathrm{~s}$ of the analysis time, $\mathrm{t}^{\mathrm{anal}}=1.65 \mathrm{~s}$, were mapped to the computational grid for $\mathrm{t}^{\text {anal }}$ as follows: the location of the measurement $\left(\psi_{n}^{\text {meas }}, s_{\text {pol }}^{\text {meas }}\right)$ was established using an EFIT-based computational grid generated for time $\mathrm{t}^{\text {meas }}$, where $\psi_{\mathrm{n}}^{\text {meas }}$ is the normalized magnetic flux coordinate $\left(\psi_{\mathrm{n}}=1\right.$ for the separatrix $)$ and $s_{\text {pol }}^{\text {meas }}$ is the poloidal projection of the distance from the target, along $\mathbf{B}$ to the measurement location; this experimental value was then assigned to the same value of $\psi_{\mathrm{n}}$ and $\mathrm{s}_{\mathrm{pol}}$ on the computational grid for $\mathrm{t}^{\mathrm{anal}}$, i.e. $\psi_{\mathrm{n}}^{\mathrm{anal}}=\psi_{\mathrm{n}}^{\text {meas }}$, $\mathrm{s}_{\mathrm{pol}}^{\text {anal }}=\mathrm{s}_{\mathrm{pol}}^{\text {meas }}$; thus the EFIT uncertainties entered the data assignment procedure twice.

The OSM analysis procedure is centrally dependant on the computational grid used and thus on EFIT and its uncertainties. In the JET OSM study [5] it was found that at the upstream probe location the OSM-calculated and measured $\mathrm{T}_{\mathrm{e}}(\mathrm{r})$ and $\mathrm{n}_{\mathrm{e}}(\mathrm{r})$ profiles were in serious disagreement if the EFIT-calculated separatrix location was accepted as being correct. When, however, the JET upstream probe data were shifted by $2 \mathrm{~cm}$ (midplane equivalent) the $T_{e}$ and $n_{e}$ profiles matched to within experimental error for most of the shots. The separatrix location defined by EFIT can vary significantly due to the effects of edge currents, current near the X-point, edge pressure gradients, etc. In this DIII-D study various settings of the EFIT parameters were tried, including with/wo edge currents. These different settings shifted the location of the outer strike point by $\sim 1 \mathrm{~cm}$. While inclusion of edge currents is known to be essential for H-modes on DIII-D, the situation for L-modes is not clear. In the end it was found that the best matches between the OSM-(target probe)-calculated $\mathrm{n}_{\mathrm{e}}(\mathrm{s} \|)$ and $\mathrm{T}_{\mathrm{e}}(\mathrm{s} \|)$ profiles in the outer divertor leg and the Thomson measurements were obtained for EFITs that did not assume edge currents and with a shift of the computational grid by $\Delta \mathrm{R}=+10 \mathrm{~mm}$ (it was simpler to shift the experimental data relative to the grid by $\Delta \mathrm{R}=-10 \mathrm{~mm}$, and this was what was done here). Comparisons of the OSM and Thomson $\mathrm{n}_{\mathrm{e}}(\mathrm{s} \|)$ and $\mathrm{T}_{\mathrm{e}}(\mathrm{s} \|)$ profiles for the first four computational "rings" (the only ones which had divertor Thomson data) are shown in Fig. 3. The Thomson measurements show significant scatter, in part because this method involves a $\sim 10 \mathrm{~ns}$ integration time and thus 
captures different phases of plasma fluctuations with each laser shot. Within this substantial scatter the OSM/probe-calculated and Thomson values appear to be in agreement. If a data shift in the opposite direction was used, e.g. by $\Delta \mathrm{R}=+10 \mathrm{~mm}$, then the OSM and Thomson match was noticeably poorer compared with that in Fig. 3. It was therefore the working hypothesis that the EFIT error for this shot/time was such as to require all divertor data to be shifted by $\Delta \mathrm{R}=-$ $10 \mathrm{~mm}$ relative to the fixed grid, and the rest of the comparisons with experiment were made on that basis.

The comparison with the photodiode $\mathrm{D}_{\alpha}$ poloidal distribution across the outer target is shown in Fig. 4. The agreement is good, essentially confirming the target probe values, particularly of $\mathrm{I}_{\text {sat }}^{+}$.

The comparison of the OSM and "Core" Thomson $n_{e}(Z)$ and $T_{e}(Z)$ profiles is shown in Fig. 5. As in the JET OSM study, unless a shift of the experimental data relative to the grid was made the profiles did not match particularly well (although significantly better than on JET). In Fig. 5 the Thomson data were shifted up by $\Delta \mathrm{Z}=+15 \mathrm{~mm}$, which gave the best profile matches — and ones largely within the scatter of the Thomson data.

A first-cut, multifluid UEDGE 6] analysis was also carried out for this shot/time, assuming $\chi_{\perp \mathrm{e}}^{\mathrm{SOL}}=\chi_{\perp \mathrm{i}}^{\mathrm{SOL}}=2.5 \mathrm{~m}^{2} / \mathrm{s}, \mathrm{D}_{\perp}^{\mathrm{SOL}}=0.625 \mathrm{~m} / \mathrm{s}$, a recycling coefficient at the walls and targets of unity, and carbon physical and chemical sputtering from the Toronto database [12]. The plasma density was set to match the experimental value upstream near the separatrix, Fig. 5. These initial UEDGE results are also shown in Figs. 3-5.

\section{Discussion and conclusions}

The OSM analysis method has been successfully applied to a more extensive set of edge measurements than has been modeled previously, encouraging further application and development of the method. The issues that remain to be addressed are substantial. 
The equilibrium reconstruction analysis completed for these discharges involved uncertainties in the separatrix locations that allow shifting experimental data relative to the computational grid. This may, however, be "sweeping under the carpet" real discrepancies. This may also be obscuring the fact that important physics, such as drift effects, needs to be included in the OSM analysis. More extensive OSM analysis work, including larger edge databases and application to other discharge types, also direction of $\mathbf{B}$, will help to address this. It is also anticipated that more evolved EFIT procedures ("kinetic EFIT") and ongoing refinements to EFIT, including the planned use of a new Li beam diagnostic, will help to better define the magnetic configuration at the edge.

Edge Thomson scattering data are a particularly important part of the DIII-D edge database. Because they sample the fluctuations in $n_{e}$ and $T_{e}$, however, they tend to be characterized by large variations and in order to make more useful comparisons to the (steady-state) OSM values, it will be necessary to analyze cases where the $\mathrm{X}$-point was not swept across the divertor Thomson location, and to average over a number of Thomson laser shots.

Further effort will be required to extend OSM analysis to the private zone and to the (generally detached) inner divertor plasma. Also lying ahead is the application of DIVIMP to the OSM-generated "plasma background", the 2D edge fields of $\mathrm{n}_{\mathrm{e}}$, etc., to analyze impurity behavior. Further work on the interface with UEDGE code analysis is required, including the use in the 2-D fluid code of transport coefficients extracted by OSM.

\section{Acknowledgment}

Work supported by the U.S. Department of Energy under Contract Nos. DE-AC0399ER54463， DE-AC04-94AL85000， W-7405-ENG-48， DE-AC05-00OR22725， DE-AC0276CH03073 and Grant No. DE-FG03-95ER54294 and ITER Canada.

\section{References}


[1] D. Reiter, J. Nucl. Mater. 196-198 (1992) 80.

[2] P.C. Stangeby and J.D. Elder, Nucl. Fusion 35 (1995) 1391.

[3] P.C. Stangeby, J.D. Elder and W. Fundamenski, et al., J. Nucl. Mater. 241-243 (1997) 358.

[4] W. Fundamenski, P.C. Stangeby and J.D Elder, J. Nucl. Mater. 266-269 (1999) 1045.

[5] S. K. Erents, P.C. Stangeby, B. LaBombard, et al., Nucl. Fusion 40 (2000) 295, 309.

[6] T.D. Rognlien, P.N. Brown, R.B. Campbell, et al., Contrib. Plasma Phys 34 (1994) 362.

[7] See references in P.C. Stangeby "The Plasma Boundary of Magnetic Confinement Devices", Institute of Physics Publishing, Bristol, 2000; chapter 5.

[8] T.N. Carlstrom, et al., Rev Sci Instrum., 63 (1992) 4901; 66 (1995) 493.

[9]. J.G. Watkins, R.A. Moyer, J.W. Cuthbertson, et al., J. Nucl. Mater. 241-243 (1997) 645.

[10] R.C. Isler, R.W. Wood, C.C. Klepper, et al., Phys Plasmas 4 (1997) 355.

[11] L.L. Lao, et al., Nucl. Fusion 30 (1990) 1035.

[12] J.W. Davis and A.A. Haasz, J. Nucl. Mater. 241-243 (1997) 37. 


\section{Figure Captions}

Fig. 1. Magnetic equilibrium for shot 86575 at $1650 \mathrm{~ms}$ showing location of edge diagnostics.

Fig. 2. $\mathrm{T}_{\mathrm{e}}$ and $\mathrm{I}_{\text {sat }}^{+}$across the outer target from the Langmuir probe array. The vertical dashed lines indicate the separatrix.

Fig. 3. (a). $\mathrm{T}_{\mathrm{e}}\left(\mathrm{s}_{\|}\right)$profiles for the first four computational "rings" (onion skins) in the SOL, which make contact with the outer target over an extent of about $1.5 \mathrm{~cm}$ starting at the outer strike point. Ring 10 is nearest the separatrix. Divertor Thomson: individual points. OSM: continuous line. UEDGE: dashed line. The OSM solutions start from the Langmuir probe values at the target (squares). All data have been shifted by $\Delta \mathrm{R}=-10 \mathrm{~mm}$ relative to the EFIT-based computational grid.

Fig. 3. (b). As Fig. 3 (a) but for $n_{e}\left(s_{\|}\right)$.

Fig. 4. $\mathrm{D}_{\alpha}$ emissivity [photons $/ \mathrm{m}^{2} / \mathrm{s}$ ] across the outer target. Experiment (filterscope): crosses. OSM: solid line. UEDGE code: dashed line.

Fig. 5. Upstream $T_{e}(Z)$ and $n_{e}(Z)$ profiles along the line of the "Core" Thomson scattering diagnostic, Fig. 1. The vertical dashed line indicates the separatrix. Thomson values: individual points. OSM: continuous line. UEDGE: dotted line. The Thomson data have been shifted by $\Delta \mathrm{Z}$ $=+15 \mathrm{~mm}$ relative to the EFIT-based grid. 


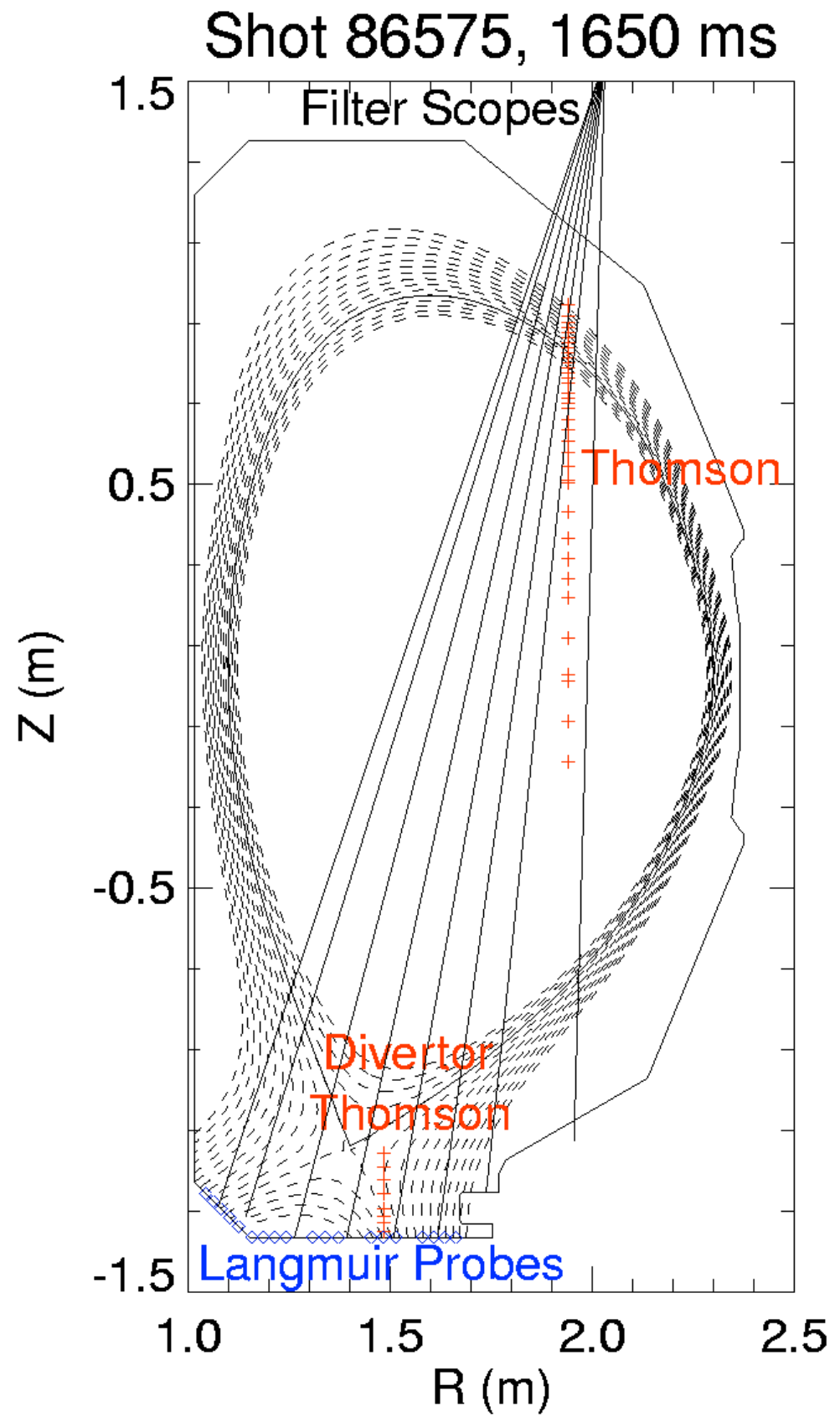

Fig. 1. 

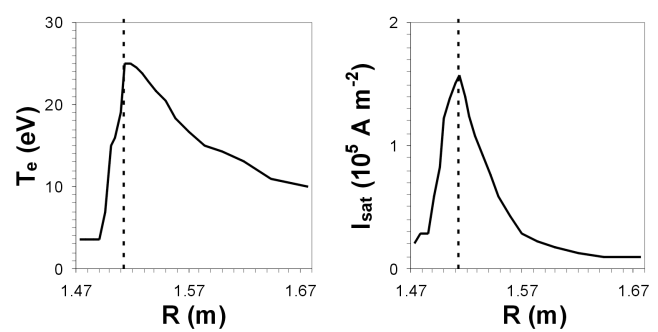

Fig.2
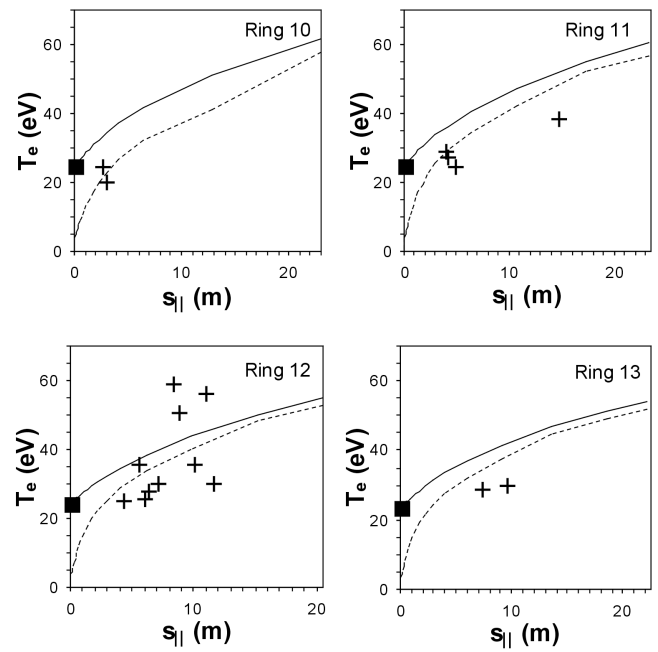

Fig. 3a
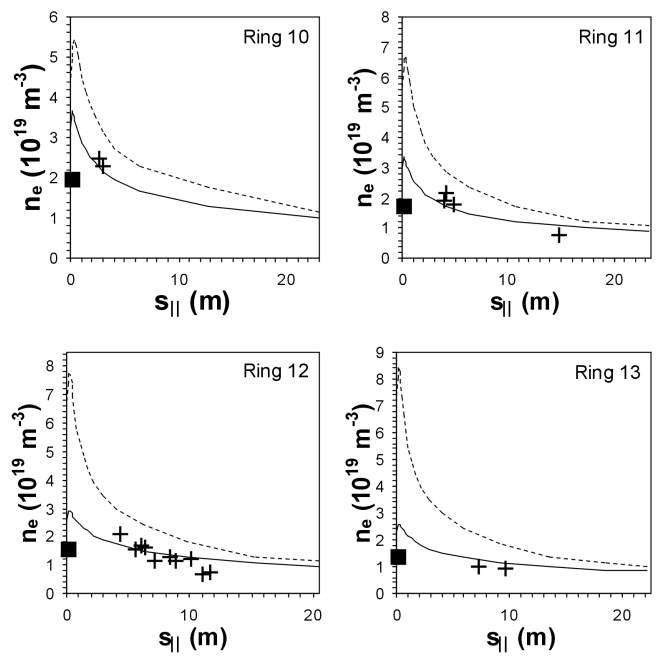

Fig 3b 


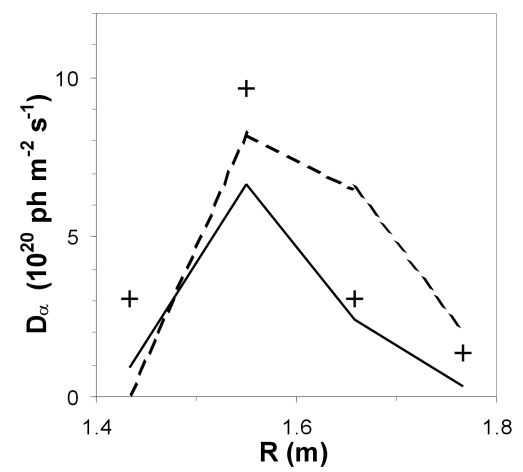

Fig. 4
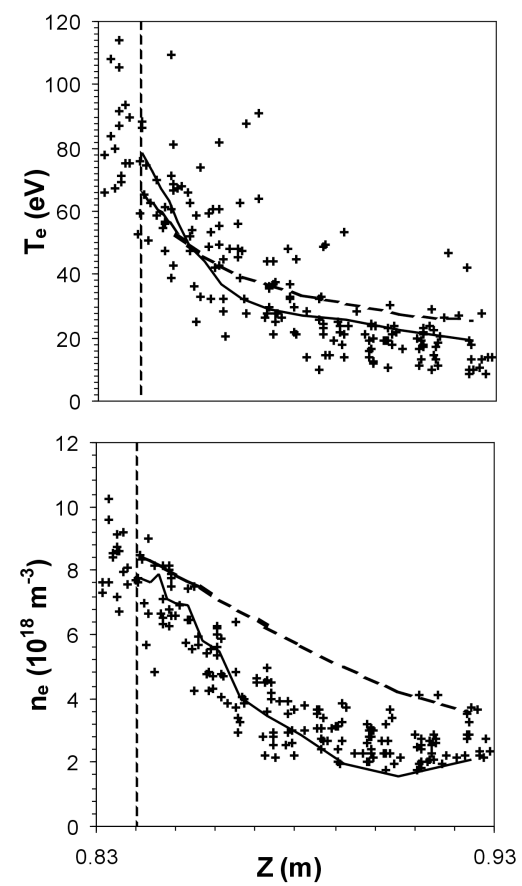

Fig. 5 